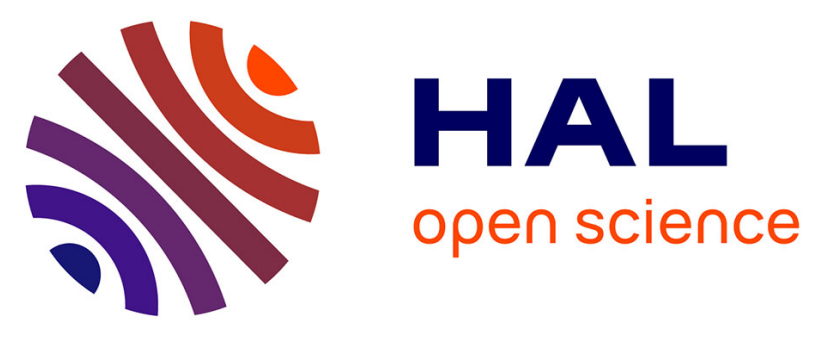

\title{
Comparison of Choi criteria and Response Evaluation Criteria in Solid Tumors (RECIST) for intrahepatic cholangiocarcinoma treated with glass-microspheres Yttrium-90 selective internal radiation therapy (SIRT)
}

Luc Beuzit, Julien Edeline, Vanessa Brun, Maxime Ronot, Anne

Guillygomarc'H, Karim Boudjema, Yves Gandon, Etienne Garin, Yan Rolland

\section{To cite this version:}

Luc Beuzit, Julien Edeline, Vanessa Brun, Maxime Ronot, Anne Guillygomarc'H, et al.. Comparison of Choi criteria and Response Evaluation Criteria in Solid Tumors (RECIST) for intrahepatic cholangiocarcinoma treated with glass-microspheres Yttrium-90 selective internal radiation therapy (SIRT). European Journal of Radiology, 2016, 85 (8), pp.1445-1452. 10.1016/j.ejrad.2016.05.020 . hal-01363197

\section{HAL Id: hal-01363197}

https://hal-univ-rennes1.archives-ouvertes.fr/hal-01363197

Submitted on 20 Oct 2016

HAL is a multi-disciplinary open access archive for the deposit and dissemination of scientific research documents, whether they are published or not. The documents may come from teaching and research institutions in France or abroad, or from public or private research centers.
L'archive ouverte pluridisciplinaire HAL, est destinée au dépôt et à la diffusion de documents scientifiques de niveau recherche, publiés ou non, émanant des établissements d'enseignement et de recherche français ou étrangers, des laboratoires publics ou privés. 
Comparison of Choi Criteria and Response Evaluation Criteria in Solid Tumors (RECIST) for

Intrahepatic Cholangiocarcinoma Treated with Glass-microspheres Yttrium-90 Selective

Internal Radiation Therapy (SIRT)

\section{Manuscript type}

Original study

\section{Authors}

Luc Beuzit ${ }^{1}, \mathrm{MD}$

Julien Edeline ${ }^{2}$, MD

Vanessa Brun ${ }^{1}$, MD

Maxime Ronot ${ }^{3}, \mathrm{MD}, \mathrm{PhD}$

Anne Guillygomarc' $h^{4}$, MD

Karim Boudjema ${ }^{5}$, MD, PhD

Yves Gandon ${ }^{1}$, MD

Etienne Garin ${ }^{6}, \mathrm{MD}, \mathrm{PhD}$

Yan Rolland ${ }^{7}, \mathrm{MD}, \mathrm{PhD}$

${ }^{1}$ Department of Radiology, Rennes University Hospital, 2 Rue Henri Le Guilloux, 35000 Rennes, France

${ }^{2}$ Department of Medical Oncology, Comprehensive Cancer Center, Institute Eugène Marquis, Rennes, France

${ }^{3}$ Department of Radiology, Beaujon University Hospital, Assistance Publique Hopitaux de Paris, Clichy, France

${ }^{4}$ Department of Hepatology, Rennes University Hospital, Rennes, France

${ }^{5}$ Department of Digestive Surgery, Rennes University Hospital, Rennes, France

${ }^{6}$ Department of Nuclear Medicine, Comprehensive Cancer Center, Institute Eugène Marquis, Rennes, France

${ }^{7}$ Department of Radiology, Comprehensive Cancer Center, Institute Eugène Marquis, Rennes, France

\section{Name and address for correspondence}

Luc Beuzit

Service de radiologie, CHU Pontchaillou

2 Rue Henri Le Guilloux, 35000 Rennes, France

luc.beuzit@chu-rennes.fr ; +33299284309 


\title{
Highlights
}

- After selective internal radiation therapy in intrahepatic cholangiocarcinoma, Choi criteria better identify patients with long survival than RECIST

- Choi criteria should be used for the assessment of treatment efficacy in this situation

\begin{abstract}
Objective: To compare Choi criteria with Response Evaluation Criteria in Solid Tumors (RECIST) for the prediction of overall survival (OS) in patients treated with glassmicrospheres, Yttrium-90 selective internal radiation therapy (SIRT) for intrahepatic cholangiocarcinoma (ICC).
\end{abstract}

Methods: Between 2010 and 2014, 45 adult patients with locally advanced ICC treated with SIRT were retrospectively analyzed. Computed Tomography scans performed before and after treatment were analyzed using both RECIST 1.1 and Choi criteria. Response was correlated with survival.

Results: Patients who achieved an objective response according to Choi had a longer OS than non-responders (median OS 19.9 months [95\% CI, 1.1-38.7 months] vs. 7.5 months if stable disease [uncountable CI] and 3 months if progressive disease [95\% CI, 0-6.2 months], logrank test: $p=.003$ ) whereas there was no significant survival difference according to the RECIST response $(p=.339)$. Among the 39 RECIST non-responding patients, those identified as responders by Choi $(n=31)$ had significantly better OS than Choi nonresponders (median OS 19.9 months (95\% CI, 5.1-34.7 months) and 5.4 months (95\% CI, 011.6 months), $p=.005)$.

Conclusions: Choi criteria appear more appropriate than RECIST to identify responders with long survival among patients who received SIRT for ICC. 


\section{Abbreviations}

Confidence interval (CI)

European Association for the Study of Liver (EASL)

Intrahepatic cholangiocarcinoma (ICC)

Objective response (OR)

Overall survival (OS)

Response evaluation criteria in solid tumors (RECIST)

Yttrium-90 glass microspheres selective internal radiotherapy (SIRT)

\section{Keywords}

- Selective Internal Radiation Therapy (SIRT)

- Cholangiocarcinoma

- Treatment Outcome

- CT

- Response Evaluation Criteria In Solid Tumors (RECIST)

- Choi Criteria 


\section{Introduction}

Intrahepatic cholangiocarcinoma (ICC) is the second most common primary hepatic malignancy, with an increasing incidence in western countries [1]. Overall, the prognosis is dismal, with 5-years overall survival (OS) rate after diagnosis around 10\% [2]. Surgery is the only curative treatment, but most patients present with unresectable disease at the time of diagnosis [3]. In this setting, microspheres yttrium-90 selective internal radiation therapy $\left({ }^{90} \mathrm{Y}\right.$ SIRT), also known as radioembolization, is being studied as a safe alternative or complement to systemic chemotherapy, with reported promising results in terms of survival and limited toxicity [4-9].

Response Evaluation Criteria in Solid Tumors (RECIST) is the standard method for the evaluation of response in solid tumors. RECIST is based on the change in size of target lesions [10]. Nevertheless, response to ${ }^{90} \mathrm{Y}$ SIRT is complex and frequently leads to necrosis without actual decrease of tumor size [11]. Therefore, RECIST may not be suitable for ICC treated with ${ }^{90} \mathrm{Y}$ SIRT, and have been shown to be poorly correlated with OS [12].

Choi criteria were initially introduced to evaluate imatinib treatment in patients with gastrointestinal stromal tumors by measuring both size and density variations in the target lesions; thus, a partial response is defined by $\geq 10 \%$ decrease in the sum of largest tumor diameter or $\geq 15 \%$ decrease in the mean of tumor density [13]. Since then, they have been evaluated for the assessment of response to various treatment modalities, including targeted therapy in hepatocellular carcinoma ([14]), and more importantly in patients treated with ${ }^{90} \mathrm{Y}$ SIRT for hepatocellular carcinoma or colorectal liver metastases [15]. They were shown to identify more responders than conventional dimension-based criteria, and to better correlate with survival. To date, no published data exist regarding their value in patients with ICC treated with ${ }^{90}$ Y SIRT. 
Therefore, the objective of this study was to compare Choi criteria with RECIST for the prediction of OS, in patients treated with ${ }^{90} \mathrm{Y}$ SIRT for ICC.

\section{Materials and Methods}

\section{Study Group}

Institutional review board approval was obtained for this monocentric retrospective study.

All patients with locally advanced ICC treated with ${ }^{90} \mathrm{Y}$ SIRT at our institution between April 2010 and February 2014 were retrospectively identified and analyzed. General exclusion criteria for ${ }^{90} \mathrm{Y}$ SIRT in our institution are Child-Pugh class B cirrhosis, total bilirubin $>35$ $\mu \mathrm{mol} / \mathrm{L}$, involvement by the tumor of more than $70 \%$ of the liver $(50 \%$ in case of cirrhosis) and performance status $>2$.

Inclusion criteria for this study were: 1 / histologically-proven ICC deemed unresectable by a multidisciplinary team meeting specialized in liver malignancies, including hepatobiliary surgeons, diagnostic and interventional radiologists, and hepatologists; 2/ at least 6 months follow-up after the treatment by ${ }^{90} \mathrm{Y}$ SIRT; and 3/ available baseline contrast-enhanced computed tomography (CT) scan obtained within 2 months prior to the first ${ }^{90} \mathrm{Y}$ SIRT injection and at least one $\mathrm{CT}$ performed up to 3 months following the treatment.

Exclusion criteria were: 1/ extra-hepatic spread (hilar lymph nodes with a short axis smaller than $15 \mathrm{~mm}$ and pulmonary nodules with a great axis smaller than $10 \mathrm{~mm}$ were considered non malignant) and 2/ a concomitant active neoplasia. Hilar cholangiocarcinomas were not excluded for ${ }^{90} \mathrm{Y}$ SIRT if a mass-forming lesion could be seen within the liver.

A total of 45 patients were enrolled (Figure 1). Patients' characteristics are summarized in Table 1. 
A total of 182 lesions (including 69 target lesions in terms of response assessment) were analyzed, corresponding to a median 2 and a mean 4 lesions per patients. ${ }^{90} \mathrm{Y}$ SIRT was performed for recurrent cholangiocarcinoma in 18 patients (40\%). A total of $8(18 \%)$ patients had cirrhosis, always classified Child-Pugh A. Forty-one patients (91\%) received prior chemotherapy, and $13(29 \%)$ had concomitant chemotherapy (i.e. started at a maximum of 3 months before ${ }^{90} \mathrm{Y}$ SIRT, and continued following ${ }^{90} \mathrm{Y}$ SIRT). Other previous treatments were chemoembolization ( 1 patient) and percutaneous radiofrequency ablation ( 2 patients), but in all cases other lesions were used for response evaluation in this study.

\section{${ }^{90}$ Y SIRT Procedure}

${ }^{90} \mathrm{Y}$ SIRT was administered according to standard procedure [16]. At the end of the diagnostic angiography, Tc-99m macroaggregated albumin was injected selectively in the right or left arterial branch in order to assess the percentage of pulmonary shunting and confirm the absence of digestive uptake, particularly gastroduodenal. ${ }^{90} \mathrm{Y}$ SIRT was performed 8 to 15 days later during a second angiography, using glass microspheres (TheraSphere ${ }^{\circledR}$; BTG, London, United Kingdom) with the aim of administering a dose of $120 \pm 20$ Gy to the injected liver volume without exceeding a dose of 30 Gy to the lungs. Calculation of the delivered dose was inferred from SPECT/CT data [17].

\section{Data Collection and Radiological Interpretation}

Baseline clinical and biological data including tumor histology, presence of cirrhosis, previous and concomitant treatments, liver function (bilirubin, aminotransferases, albumin and prothrombin time) were extracted from medical charts. Dosimetric analyses of ${ }^{90} \mathrm{Y}$ SIRT procedures were also reviewed: injected activity; average absorbed dose in the tumor and in the healthy liver; and pulmonary shunt. 
All images were obtained on a multi-detector-row helical CT scanner (Somatom Definition AS, Siemens, Erlangen, Germany), including at least an unenhanced acquisition on the liver and a contrast-enhanced acquisition at the portal ( 70 seconds) phase covering the thorax and the abdomen. A 2-mL/kg injection of iodine contrast media $(300 \mathrm{mg} / \mathrm{mL})$ with an average flow rate of $3 \mathrm{~mL} / \mathrm{second}$ was performed. Slice thickness was $1.5 \mathrm{~mm}$, matrix was $512 \mathrm{X} 512$.

The baseline and two first post-treatment $\mathrm{CT}$ were retrospectively reviewed by two radiologists $\left(\mathrm{LB}, 4^{\text {th }}\right.$ year radiology resident; $\mathrm{VB}$, senior radiologist with 7 years of experience in liver imaging) blinded to the clinical and follow-up data. According to RECIST 1.1 recommendations, at baseline, target lesions had to be at least $\geq 1 \mathrm{~cm}$ for the largest diameter and non-target lesions could have a largest diameter of $<1 \mathrm{~cm}$. A maximum of two target lesions per organ and five lesions in total were selected [10]. For each target lesion, the maximum diameter, and tumor density were measured. Tumor density was evaluated at the portal phase by selecting a polygonal region of interest encompassing the entire target lesions on the slice of the measurement of the largest diameter [18] (Figure 2). Although ICC may enhance on delayed acquisitions, the portal phase was used in agreement with the original method described by Choi and al. [13]. The average density of target lesions was calculated. Response was then separately assessed according to RECIST and Choi criteria in a nonblinded fashion and the best response for each criterion among the two post treatment CT was recorded. A consensus was reached in case of disagreement. RECIST and Choi criteria are summarized in supplementary data.

A decrease in density $\geq 15 \%$ was accepted as a criterion of partial response according to Choi only if the absolute density change would account for at least 10 Hounsfield Units [18]. In case of multiple ${ }^{90} \mathrm{Y}$ SIRT injections, the response was evaluated on the treated lesions at the time of evaluation. Objective response (OR) was defined as the sum of complete and partial 
responses. We also recorded the best variations (maximal decrease or minimal increase) in the sum of target lesions diameters and their average density for each patient.

\section{Statistical Analysis}

Data were expressed as median and range, and count and rate. Continuous data were compared using the non-parametric Mann-Whitney U test, and categorical data were compared using the chi-square test or the exact Fisher's test, as appropriate. Inter-reader agreement was assessed with the Cohen's kappa statistics. Survival analyses started on the date of the first ${ }^{90} \mathrm{Y}$ SIRT injection. Overall survival analysis ended at the time of death or was censored at the time of the last follow-up visit. Progression-free survival ended at the time of death or progressive disease according to RECIST or was censored at the time of the last follow-up visit. Survival data were analyzed by using the Kaplan-Meier method with the log-rank test, and with a Cox regression model with univariate and multivariate analysis. All variable with a $p$ value $<0.1$ in univariate analysis were entered in the multivariate analysis, using a backward likelihood ratio method. A $p$ value $<0.05$ was considered significant. Analysis was performed using the SPSS software package (version 19.0; SPSS, Inc., Chicago, IL).

\section{Results}

\section{Radiological Response}

The median time between the baseline pre-treatment scan and ${ }^{90} \mathrm{Y}$ SIRT was 17 days (range 1 - 44 days); it was 49 days (range 27 - 77 days) between ${ }^{90} \mathrm{Y}$ SIRT and the first post-treatment scan. Thirty-five patients $(78 \%)$ had two or more post-treatment scans. The median time between ${ }^{90} \mathrm{Y}$ SIRT and the second post-treatment scan was 133 days (range 83 - 343 days). 
Best responses as assessed by RECIST and Choi criteria are reported in Table 2 . The OR rates according to RECIST and Choi criteria were 13\% (6 of 45 patients) and $82 \%$ (37 of 45), respectively. Overall, discordance between the two methods of response assessment was observed for 31 patients (69\%), resulting in a kappa of $0.06 \pm 0.03$.

At baseline, the median size of the biggest tumor was $60 \mathrm{~mm}$ (range, $18-136 \mathrm{~mm}$ ), with a median attenuation of 73 Hounsfield Units $(47-145 \mathrm{HU})$. The maximal variations in the sum of the greatest target lesions diameters and in their average density are presented in Figure 3. There was no correlation between changes in size and changes in density (Pearson's correlation coefficient $r=0.09, p=0.56$ ).

At the time of analysis, progression was observed in 25 patients (56\%). Progression was defined as the appearance of new lesions in 24 patients and by an unequivocal progression of non-target lesions in 1 patient. Progression involved the liver for 15 patients, including the parenchyma treated by the injection of ${ }^{90} \mathrm{Y}$ SIRT for 9 patients, amongst whom 8 also had an extra hepatic progression. Progression only in the liver treated by ${ }^{90} \mathrm{Y}$ SIRT was thus seen only in $1(2 \%)$ patient. Progression involved extra hepatic sites in 21 patients, involving the lungs for 14 patients, the peritoneum for 6 patients, distal lymph nodes for 3 patients and bones for 2 patients.

Only one patient experienced severe toxicity after ${ }^{90} \mathrm{Y}$ SIRT and was hospitalized for hepatic encephalopathy. This patient had a partial response according to Choi criteria, stable disease according to RECIST, and died 5 months after treatment. 


\section{Survival Analysis According to Radiological Response}

For the entire cohort, the median OS was 19.0 months $(95 \%$ confidence interval $[\mathrm{CI}], 8.6-$ 29.3 months); the 1- and 2-year OS rates were $54.0 \%$ and $40.7 \%$, respectively. Treatment allowed downstaging and resection in 9 patients $(20 \%)$.

The Kaplan-Meier estimates of OS according to the response by RECIST and Choi criteria are presented in Figure 4.

The OS of responders according to Choi criteria was significantly longer than that of patients with stable or progressive disease, with medians of 19.9 months (95\% CI, $1.1-38.7$ months), 7.5 months (95\% CI : non evaluable) and 5.4 months (95\% CI, $0-6.2$ months), respectively (log-rank test: $p=0.003)$. There was no difference in OS between patients who achieved an OR as assessed by RECIST and those who did not (log-rank test: $p=0.339)$. The progressionfree survival of patients who achieved an OR according to Choi criteria was significantly longer than that of non-responders, with a median 9.5 months (95\% CI, $7.9-11.1$ months), and 2.0 months (95\% CI, $1.3-2.7$ months), respectively (log-rank test: $p<0.001)$.

Finally, in the 39 non-responding patients according to RECIST, an objective response according to Choi criteria was associated with a better OS (Figure 5), with a median 19.9 months (95\% CI, 5.1 - 34.7 months) and 5.4 months (95\% CI, 0 - 11.6 months) in responders $(\mathrm{n}=31)$ and non-responders $(\mathrm{n}=8)$, respectively (log-rank test: $p=.005)$.

The results of univariate and multivariate analysis of survival are given in Table 3. If forced into the multivariate model with Choi, RECIST response was not associated with survival.

\section{Inter-observer agreement}

The kappa for inter-observer agreement was $0.71 \pm 0.11$ for RECIST, $0.75 \pm 0.11$ for Choi criteria. Disagreements in Choi criteria evaluation were observed in 4 patients (9\%), due to discrepancies in density variation measurement $(n=1)$, the interpretation of the appearance of 
ascites and pleural effusion $(n=1)$, and the appearance of small hypodense lesions on the post therapeutic CT scan interpreted by one reader as evidence of progression due to new lesion, and by the other as response with necrosis of pre-existing lesion $(n=2)$. Disagreements in RECIST evaluation were observed in 6 patients $(13 \%)$, due to differences in the measurement of size variation $(n=3)$ or by the interpretation of non-target lesions and new lesions (same cases as for Choi criteria).

\section{Discussion}

In this retrospective study of patients treated by ${ }^{90} \mathrm{Y}$ SIRT for ICC, we sought to compare Choi criteria with RECIST in terms of predicting OS. We found that patients who achieved an OR according to Choi criteria had improved OS and progression-free survival as compared to non-responding patients, whereas there was no significant difference in the survival of responding and non-responding patients according to RECIST. Moreover, among the majority of patients classified as non-responders according to RECIST, the use of Choi criteria differentiated a subset of patients that benefited from ${ }^{90} \mathrm{Y}$ SIRT.

These results can be explained by the inability of RECIST to assess the necrotic effect of ${ }^{90} \mathrm{Y}$ SIRT. Tumor response assessment based on change in size may be appropriate for treatments that result in significant tumor shrinkage; however, ${ }^{90} \mathrm{Y}$ SIRT may cause predominant tissue necrosis [15]. Necrosis is related to avascular tissue, depicted on imaging as a lack on contrast uptake. This would be translated first by a decrease in tissue density, and only subsequently by a decrease in tumor size. This explains why variations in density were more marked in this study. In fact, decrease in greatest tumor diameters were also frequently noted, but in the majority of the cases, such decrease of size was lower than the $-30 \%$ threshold for an OR according to RECIST. As a consequence, the Choi criteria, evaluating both size and density, performed better in identifying the subset of patients with a better prognosis. 
We did not observe any case of complete response with both RECIST and Choi criteria, which is not surprising since it would have implied the total disappearance of all lesions, a response that is not expected at an early stage after SIRT. Only 6 among the 37 patients with partial response according to Choi criteria later experienced progression on the treated lesion.

To our knowledge, no study examining Choi criteria assessment of response to ${ }^{90} \mathrm{Y}$ SIRT in cholangiocarcinoma has been published to date. However, another study using alternative size-based response criteria (namely modified RECIST or the European Association for the Study of Liver (EASL)) have shown that enhancement of lesions was a valid surrogate for tissue viability in ICC treated by ${ }^{90} \mathrm{Y}$ SIRT [12]. Authors showed a significant lower OR rates according to RECIST (6.2\%) when compared to both mRECIST (50\%) and EASL criteria (56.2\%). Compared to Choi criteria, mRECIST and EASL criteria have the advantage of being transposable from computed tomography to magnetic resonance imaging. On the other hand, they were introduced for hypervascular lesions, thus needing an adaptation to delayedphase imaging in cholangiocarcinoma [12]. SIRT may also induce a homogenous decrease in the density of the whole lesion, which justifies the use of a global measurement of density rather than a unidimensional measurement of the assumed viable part of the lesion. Moreover, EASL criteria only evaluate the local response to treatment [19] whereas we observed that progression was mostly extra hepatic.

Most of the discrepancies observed between the two observers were due to the appearance of small hypodense lesions on the post therapeutic CT scan whereas the density of target lesions had decreased; after consensus, these lesions were considered as small isodense lesions on the baseline scan that had been revealed by the necrosis following ${ }^{90} \mathrm{Y}$ SIRT. It is likely that these lesions would have been seen on a baseline MR imaging. However, evaluation of extrahepatic spread is crucial in this context, as illustrated by the high number of patients experiencing extra-hepatic progression (21 out of 25 progressing patients). The evaluation of 
patients who received several injections of ${ }^{90} \mathrm{Y}$ SIRT was complex. Since this strategy involved an evaluation of tumor response and induction chemotherapy between two injections, several months could pass between the first and last injections. Yet, we saw that the time to progression was quite short in this population and the assessment of response after the first injection rather than at the end of treatment seems valid.

Limitations of the present study included its monocentric and retrospective design, and as a consequence the varying delay between evaluation scans which prevented any analysis of the optimal time for assessment of response to ${ }^{90} \mathrm{Y}$ SIRT. The 49 days median period between the first ${ }^{90} \mathrm{Y}$ injection and the first post-treatment scan was shorter than the average delay of 3 months chosen to assess radiological response in similar studies [8]; this attitude is routinely performed in our institution for early evaluation of innovative treatment [10]. The use of Choi criteria for the evaluation of ${ }^{90} \mathrm{Y}$ SIRT-induced responses in ICC raises several concerns regarding Choi criteria. Choice of target lesion, optimal scanning time, size and position of ROI may differ according to the readers, leading to lower reproducibility [20]. This is partially due to the few initial comprehensive practical guidelines regarding application [13]. We followed the RECIST 1.1 guidelines regarding the choice of target lesions and size measurements [10]. Regarding the density measurement, we chose a widespread, simple and reproducible method by performing an average of the densities in a ROI encompassing the whole lesion [18]. Finally, as a relatively hypodense lesion at baseline, measurements of density variations in ICC may be less reliable than in hypervascular lesions such as gastrointestinal stromal tumors or hepatocellular carcinoma, since a small absolute decrease of density may lead to a high relative variation. This is why we followed the recommendation of the German GIST imaging working group and added a minimal 10 Hounsfield Units absolute decrease to the $15 \%$ relative decrease in density to validate a PR [18]. 
In conclusion, Choi criteria seem more appropriate than RECIST for the identification of patients with ICC who benefit from ${ }^{90} \mathrm{Y}$ SIRT. They may be used for the assessment of treatment efficacy.

\section{Disclosure paragraph:}

The authors of this manuscript declare relationships with the following companies: Etienne GARIN and Yan ROLLAND - Consultant, BTG International Ltd

The authors state that this work has not received any funding.

Institutional Review Board approval was obtained. 


\section{References}

1. Khan SA, Toledano MB, Taylor-Robinson SD (2008) Epidemiology, risk factors, and pathogenesis of cholangiocarcinoma. HPB 10:77-82.

2. Everhart JE, Ruhl CE (2009) Burden of Digestive Diseases in the United States Part III: Liver, Biliary Tract, and Pancreas. Gastroenterology 136:1134-1144.

3. Dodson RM, Weiss MJ, Cosgrove D, et al. (2013) Intrahepatic Cholangiocarcinoma: Management Options and Emerging Therapies. J Am Coll Surg 217:736-750.

4. Mouli S, Memon K, Baker T, et al. (2013) Yttrium-90 Radioembolization for Intrahepatic Cholangiocarcinoma: Safety, Response, and Survival Analysis. J Vasc Interv Radiol 24:1227-1234.

5. Rafi S, Piduru SM, El-Rayes B, et al. (2013) Yttrium-90 radioembolization for unresectable standard-chemorefractory intrahepatic cholangiocarcinoma: survival, efficacy, and safety study. Cardiovasc Intervent Radiol 36:440-448.

6. Hoffmann R-T, Paprottka PM, Schön A, et al. (2012) Transarterial hepatic yttrium-90 radioembolization in patients with unresectable intrahepatic cholangiocarcinoma: factors associated with prolonged survival. Cardiovasc Intervent Radiol 35:105-116.

7. Saxena A, Bester L, Chua TC, et al. (2010) Yttrium-90 radiotherapy for unresectable intrahepatic cholangiocarcinoma: a preliminary assessment of this novel treatment option. Ann Surg Oncol 17:484-491.

8. Al-Adra DP, Gill RS, Axford SJ, et al. (2015) Treatment of unresectable intrahepatic cholangiocarcinoma with yttrium-90 radioembolization: a systematic review and pooled analysis. Eur J Surg Oncol 41:120-127.

9. Edeline J, Du FL, Rayar M, et al. (2015) Glass Microspheres 90Y Selective Internal Radiation Therapy and Chemotherapy as First-Line Treatment of Intrahepatic Cholangiocarcinoma. Clin Nucl Med. doi: 10.1097/RLU.0000000000000904

10. Eisenhauer EA, Therasse P, Bogaerts J, et al. (2009) New response evaluation criteria in solid tumours: Revised RECIST guideline (version 1.1). Eur J Cancer 45:228-247.

11. Ibrahim SM, Nikolaidis P, Miller FH, et al. (2009) Radiologic findings following Y90 radioembolization for primary liver malignancies. Abdom Imaging 34:566-581.

12. Camacho JC, Kokabi N, Xing M, et al. (2014) Modified Response Evaluation Criteria in Solid Tumors and European Association for the Study of the Liver Criteria Using Delayed-Phase Imaging at an Early Time Point Predict Survival in Patients with Unresectable Intrahepatic Cholangiocarcinoma following Yttrium-90 Radioembolization. J Vasc Interv Radiol 25:256-265.

13. Choi H, Charnsangavej C, de Castro Faria S, et al. (2004) CT evaluation of the response of gastrointestinal stromal tumors after imatinib mesylate treatment: a quantitative analysis correlated with FDG PET findings. Am J Roentgenol 183:1619-1628. 
14. Ronot M, Bouattour M, Wassermann J, et al. (2014) Alternative Response Criteria (Choi, European association for the study of the liver, and modified Response Evaluation Criteria in Solid Tumors [RECIST]) Versus RECIST 1.1 in patients with advanced hepatocellular carcinoma treated with sorafenib. The Oncologist 19:394-402.

15. Weng Z, Ertle J, Zheng S, et al. (2013) Choi criteria are superior in evaluating tumor response in patients treated with transarterial radioembolization for hepatocellular carcinoma. Oncol Lett 6:1707-1712.

16. Salem R, Lewandowski RJ, Gates VL, et al. (2011) Research Reporting Standards for Radioembolization of Hepatic Malignancies. J Vasc Interv Radiol 22:265-278.

17. Garin E, Lenoir L, Rolland Y, et al. (2011) Effectiveness of quantitative MAA SPECT/CT for the definition of vascularized hepatic volume and dosimetric approach: phantom validation and clinical preliminary results in patients with complex hepatic vascularization treated with yttrium-90-labeled microspheres. Nucl Med Commun $32: 1245-1255$.

18. Kalkmann J, Zeile M, Antoch G, et al. (2012) Consensus report on the radiological management of patients with gastrointestinal stromal tumours (GIST): recommendations of the German GIST Imaging Working Group. Cancer Imaging 12:126-135.

19. Bruix J, Sherman M, Llovet JM, et al. (2001) Clinical management of hepatocellular carcinoma. Conclusions of the Barcelona-2000 EASL conference. European Association for the Study of the Liver. J Hepatol 35:421-430.

20. Goh V, Halligan S, Gharpuray A, et al. (2008) Quantitative assessment of colorectal cancer tumor vascular parameters by using perfusion $\mathrm{CT}$ : influence of tumor region of interest. Radiology 247:726-732. 


\section{Table and figure legends}

Figure 1: consort diagram. ICC: intrahepatic cholangiocarcinoma; pt: patient

Figure 2: example of tumor response evaluation according to RECIST and Choi criteria in a 65 y.o. female with ICC. A ROI surrounding the whole lesion was drawn on the slice concerned by the greatest diameter measurement on baseline (a) and post treatment (b) scans. Concerning this patient, there was no significant modification of tumor size (-9\%, SD according to RECIST) but more than $15 \%$ decrease in tumor density ( $-32 \%$, OR according to Choi criteria). This patient was alive at the end of the study, with a 40 months follow-up following ${ }^{90} \mathrm{Y}$ SIRT. The progression-free survival was 18.6 months (pulmonary metastasis treated with stereotactic radiation).

Figure 3: Waterfall plot illustrating the best variations of target lesions diameters (black columns) and densities (grey columns), represented separately (a, b) and side-by-side (c) for each patient. The solid line represents the $-30 \%$ threshold corresponding to partial response according to the RECIST criteria, the dashed line represents the $-15 \%$ threshold corresponding to partial response in terms of density according to Choi criteria.

Figure 4: Kaplan-Meier estimates of overall survival according to the response evaluated by RECIST (a) and Choi criteria (b). Responders by Choi criteria (green solid line) display significantly higher survival (log-rank test) compared with non-responders (blue dotted line) whereas the difference was not statistically significant with RECIST.

Figure 5: Kaplan-Meier estimates of overall survival of the subset of 39 RECIST nonresponding patients (grey dotted line), according to the response evaluated by Choi criteria. Responders by Choi criteria (green solid line) display significantly higher survival (log-rank test) compared with non-responders (blue dotted line) 
52 pts with ICC treated with ${ }^{90} \mathrm{Y}-\mathrm{RE}$ in our institution

4 pts had metastases

$1 \mathrm{pt}$ had concomitant neoplasia

47 pts with ICC without extra-hepatic spread

2 pts without post-treatment CT scan $1 \mathrm{pt}$ died before evaluation $1 \mathrm{pt}$ moved abroad

45 pts included 

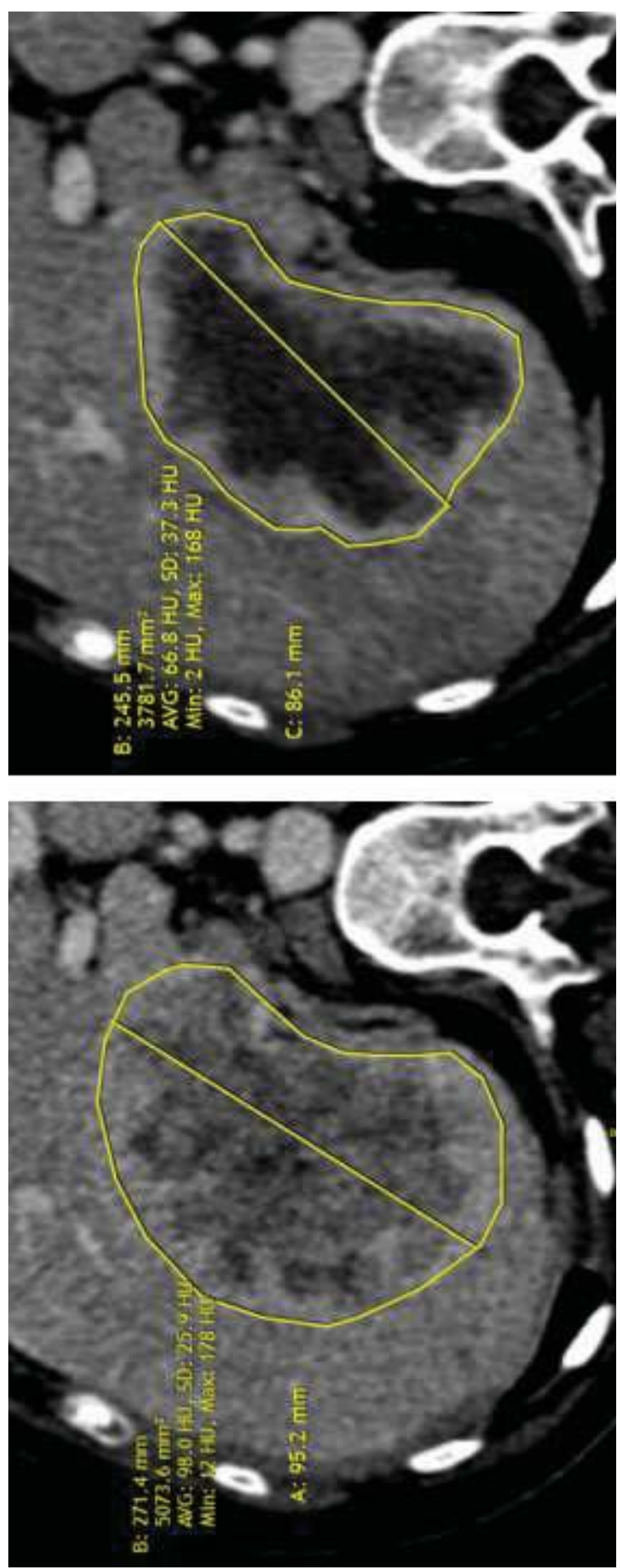


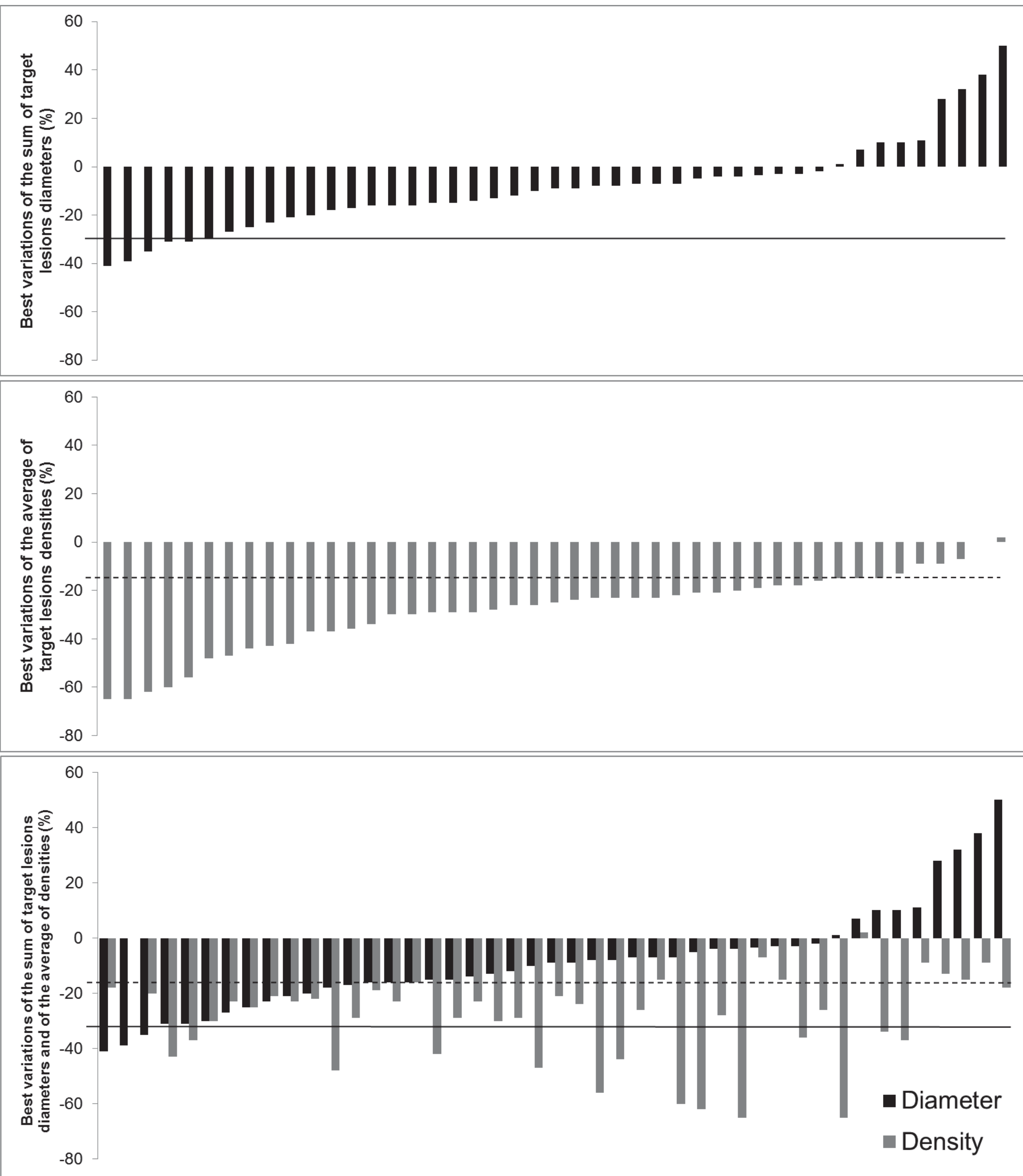




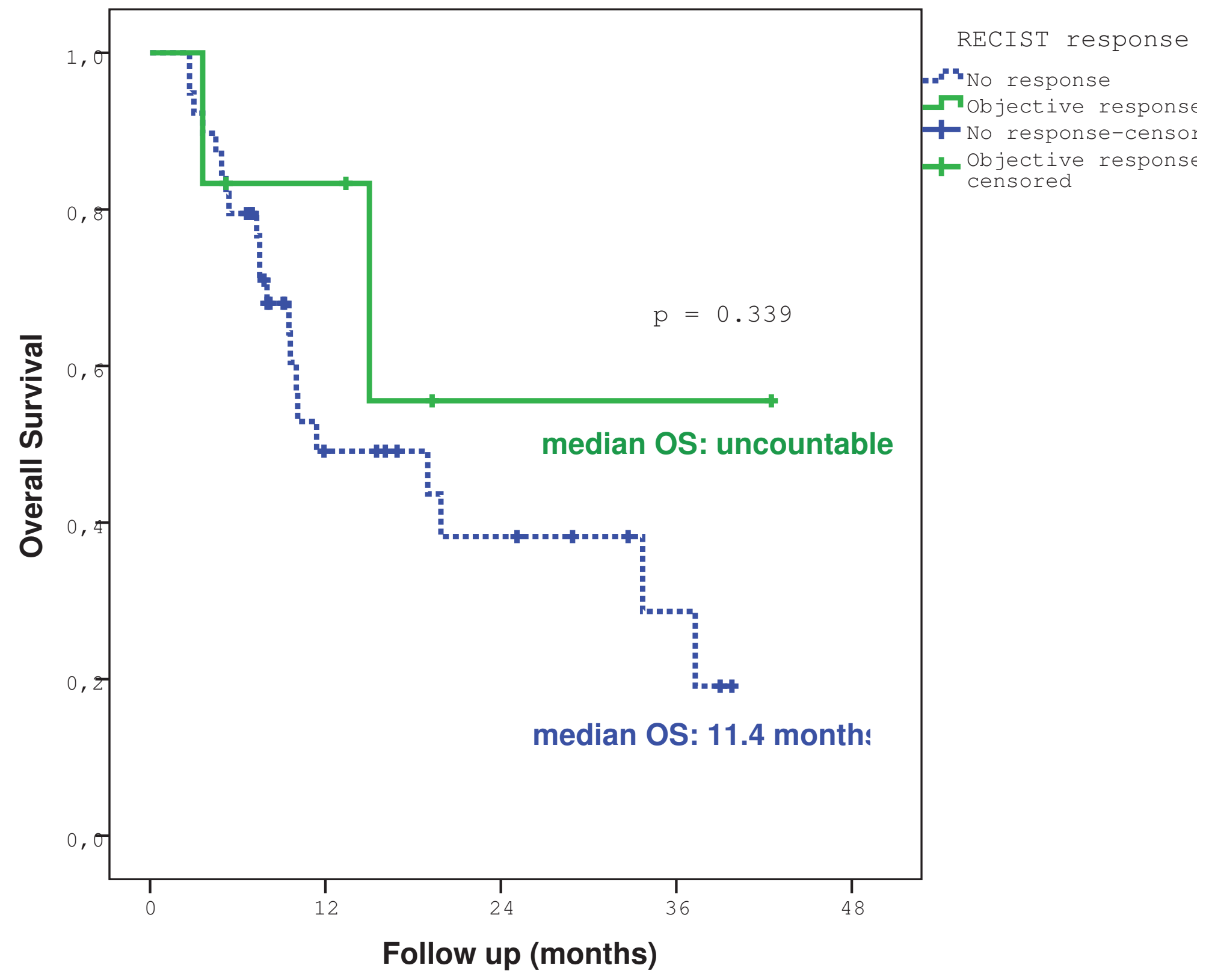




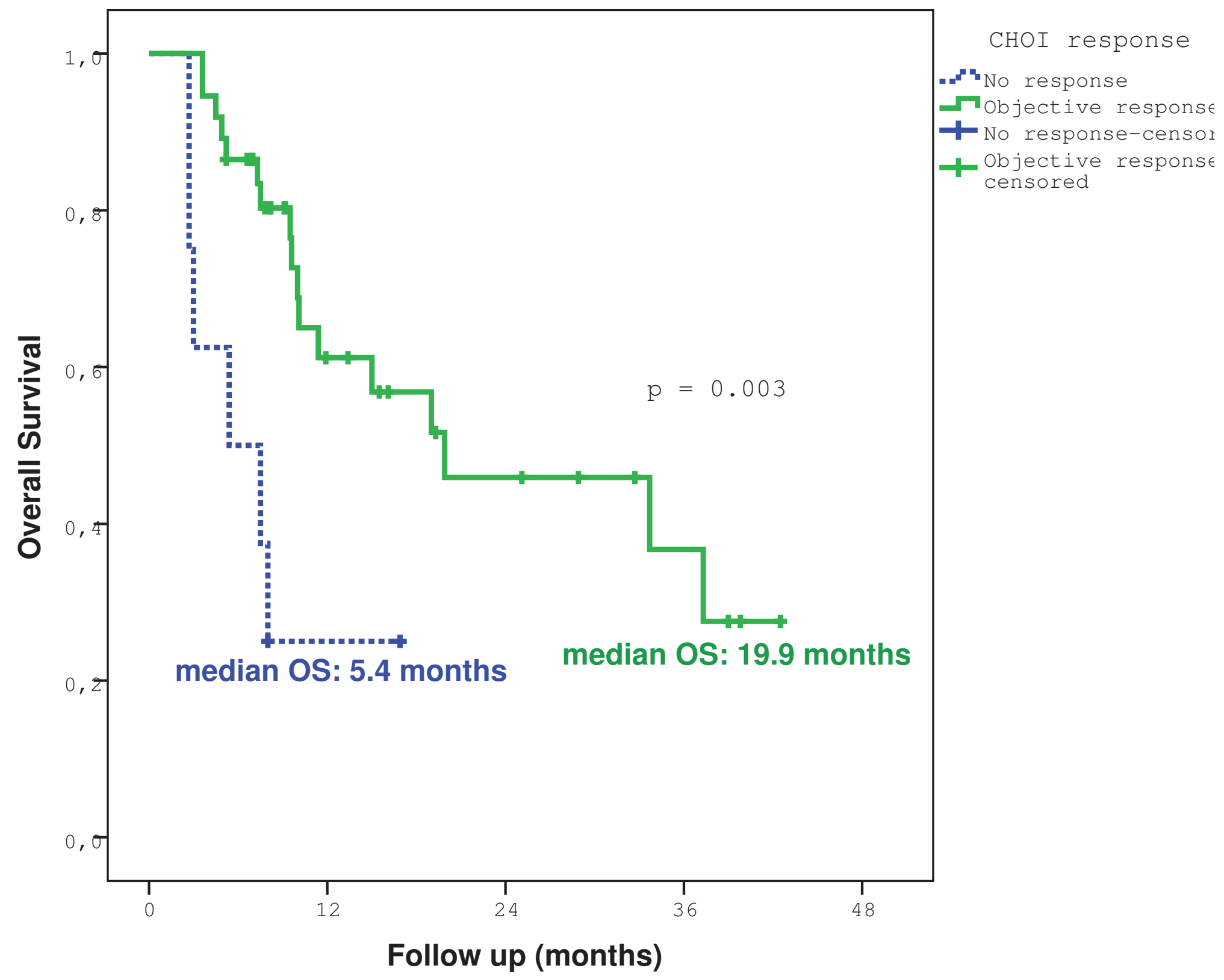




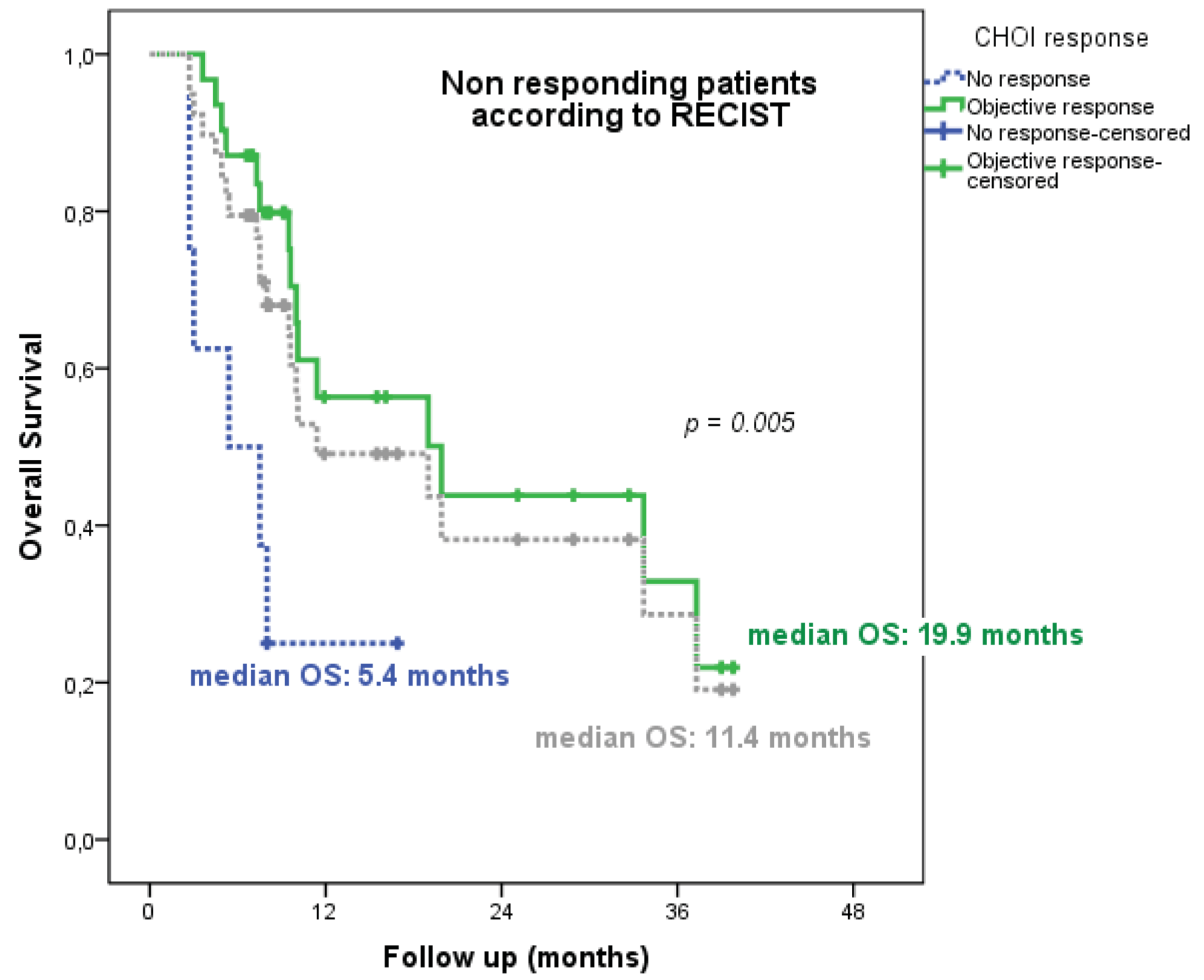


Table 1: Characteristics of the cohort. ALT: Alanine Transferase, AST: Aspartate

Transferase, ECOG: Eastern Cooperative Oncology Group, PT: Prothrombin Time, SIRT:

Selective Internal Radiation Therapy

\begin{tabular}{|c|c|}
\hline \multicolumn{2}{|l|}{ Age at diagnosis (years) } \\
\hline Median & 64 \\
\hline Range & $29-80$ \\
\hline \multicolumn{2}{|l|}{ Sex } \\
\hline Male & $24(53 \%)$ \\
\hline Female & $21(47 \%)$ \\
\hline \multicolumn{2}{|l|}{ ECOG Performance Status } \\
\hline 0 & $25(56 \%)$ \\
\hline $1-2$ & $20(44 \%)$ \\
\hline Cirrhosis & $8(18 \%)$ \\
\hline Other treatment before (or concomitant to) ${ }^{90} \mathrm{Y}$ SIRT & $42(93 \%)$ \\
\hline \multicolumn{2}{|l|}{ No. of tumors } \\
\hline$\leq 3$ & $26(58 \%)$ \\
\hline$>3$ & $19(42 \%)$ \\
\hline \multicolumn{2}{|l|}{ Largest tumor size $(\mathrm{cm})$} \\
\hline Median & 6.0 \\
\hline Range & $1.8-13.6$ \\
\hline \multicolumn{2}{|l|}{ No. ${ }^{90} \mathrm{Y}$ treatment sessions } \\
\hline 1 & $33(74 \%)$ \\
\hline 2 & $10(22 \%)$ \\
\hline 3 & $2(4 \%)$ \\
\hline \multicolumn{2}{|l|}{ Bilirubin $(\mu \mathrm{mol} / \mathrm{L})$} \\
\hline Median & 11.1 \\
\hline Range & $2.1-29.8$ \\
\hline \multicolumn{2}{|l|}{$\mathrm{AST}(\mathrm{U} / \mathrm{L})$} \\
\hline Median & 41 \\
\hline Range & $18-138$ \\
\hline \multicolumn{2}{|l|}{ ALT (U/L) } \\
\hline Median & 31 \\
\hline Range & $7-171$ \\
\hline \multicolumn{2}{|l|}{ PT ( $\%$ from control) } \\
\hline Median & 95 \\
\hline Range & $74-100$ \\
\hline \multicolumn{2}{|l|}{ Albumin (g/L) } \\
\hline Median & 42 \\
\hline Range & $29-48$ \\
\hline
\end{tabular}


Table 2: response evaluation according to RECIST and Choi criteria. PR = partial response; $\mathrm{SD}=$ stable disease $; \mathrm{PD}=$ progressive disease.

\begin{tabular}{|c|c|c|c|c|c|}
\hline & \multicolumn{3}{|c|}{ Choi } & \multirow{2}{*}{ Total } \\
\hline & & PR & SD & PD & \\
\hline \multirow{6}{*}{ RECIST } & \multirow{2}{*}{ PR } & 6 & 0 & 0 & 6 \\
\hline & & $13 \%$ & $0 \%$ & $0 \%$ & $13 \%$ \\
\hline & \multirow{2}{*}{ SD } & 30 & 2 & 0 & 32 \\
\hline & & $67 \%$ & $4 \%$ & $0 \%$ & $71 \%$ \\
\hline & \multirow{2}{*}{ PD } & 1 & 0 & 6 & 7 \\
\hline & & $2 \%$ & $0 \%$ & $13 \%$ & $16 \%$ \\
\hline \multirow{2}{*}{\multicolumn{2}{|c|}{ Total }} & 37 & 2 & 6 & 45 \\
\hline & & $82 \%$ & $4 \%$ & $13 \%$ & $100 \%$ \\
\hline
\end{tabular}


Table 3: analysis of survival with a Cox regression model with univariate and multivariate analysis. All variable with a $p$ value $<0.1$ in univariate analysis were entered in the multivariate analysis, using a backward likelihood ratio method.

\begin{tabular}{|c|c|c|c|c|}
\hline \multirow[t]{2}{*}{ Parameter } & \multicolumn{2}{|l|}{ Univariate } & \multicolumn{2}{|c|}{ Multivariate $(\mathrm{n}=44)$} \\
\hline & Hazard ratio & $p$ & Hazard ratio & $p$ \\
\hline $\begin{array}{l}\text { Choi (responder vs non- } \\
\text { responder) }\end{array}$ & $0.25(0.09-0.68)$ & 0.007 & $0.19(0.06-0.55)$ & 0.002 \\
\hline $\begin{array}{l}\text { RECIST (responder vs non- } \\
\text { responder) }\end{array}$ & $0.50(0.12-2.14)$ & 0.35 & & \\
\hline Age (continuous) & $1.03(0.99-1.07)$ & 0.078 & $1.05(1.01-1.09)$ & 0.013 \\
\hline Gender (female vs male) & $0.87(0.38-1.98)$ & 0.74 & & \\
\hline Performance Status (1 vs 0) & $1.77(0.76-4.11)$ & 0.18 & & \\
\hline Cirrhosis (yes vs no) & $1.10(0.37-3.26)$ & 0.87 & & \\
\hline $\begin{array}{l}\text { Number of lesions } \\
\text { (continuous) }\end{array}$ & $0.95(0.84-1.07)$ & 0.36 & & \\
\hline Tumor size (continuous) & $1.00(0.99-1.01)$ & 0.92 & & \\
\hline Albumin (continuous) & $0.86(0.77-0.97)$ & 0.013 & $0.89(0.79-1.01)$ & 0.064 \\
\hline Bilirubin (continuous) & $0.91(0.83-0.99)$ & 0.034 & $0.88(0.79-0.98)$ & 0.019 \\
\hline CA19.9 (continuous) & $1.00(1.00-1.00)$ & 0.12 & & \\
\hline
\end{tabular}

